\title{
An Examination of the Effects of Be Grain Size.
}

\author{
R. Cook
}

November 21, 2000

U.S. Department of Energy

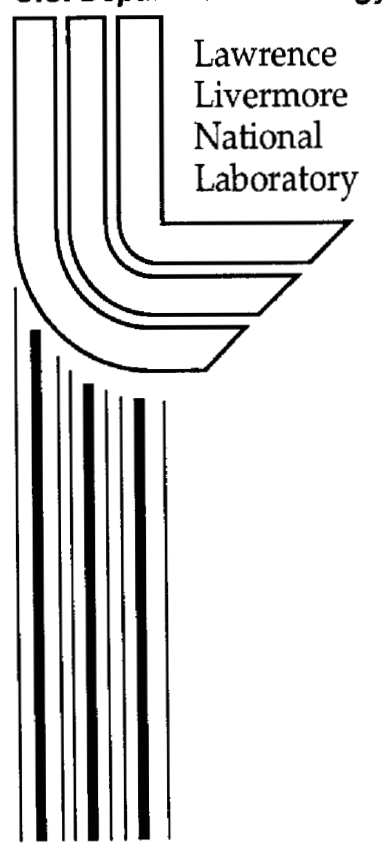




\section{DISCLAIMER}

This document was prepared as an account of work sponsored by an agency of the United States Government. Neither the United States Government nor the University of California nor any of their employees, makes any warranty, express or implied, or assumes any legal liability or responsibility for the accuracy, completeness, or usefulness of any information, apparatus, product, or process disclosed, or represents that its use would not infringe privately owned rights. Reference herein to any specific commercial product, process, or service by trade name, trademark, manufacturer, or otherwise, does not necessarily constitute or imply its endorsement, recommendation, or favoring by the United States Government or the University of California. The views and opinions of authors expressed herein do not necessarily state or reflect those of the United States Government or the University of California, and shall not be used for advertising or product endorsement purposes.

This work was performed under the auspices of the U. S. Department of Energy by the University of California, Lawrence Livermore National Laboratory under Contract No. W-7405-Eng-48.

This report has been reproduced directly from the best available copy.

Available electronically at http://www.doc.gov/bridge

Available for a processing fee to U.S. Department of Energy

And its contractors in paper from

U.S. Department of Energy

Office of Scientific and Technical Information

P.O. Box 62

Oak Ridge, TN 37831-0062

Telephone: (865) 576-8401

Facsimile: (865) 576-5728

E-mail: reports@adonis.osti.gov

Available for the sale to the public from

U.S. Department of Commerce

National Technical Information Service

5285 Port Royal Road

Springfield, VA 22161

Telephone: (800) 553-6847

Facsimile: (703) 605-6900

E-mail: orders@ntis.fedworld.gov

Online ordering: http://www.ntis.gov/ordering.htm

OR

Lawrence Livermore National Laboratory

Technical Information Department's Digital Library

http:/ / www.llnl.gov/tid/Library.html 


\section{Target Area Technologies Program}

Mail Station L-481

Ext: $2-3117$

November 21, 2000

To: Distribution

From: $\quad$ Bob Cook

Subject: An examination of the effects of Be grain size.

\section{Introduction.}

During the WBS4 review almost a year ago in January, and in more recent discussions of needed capsule characterization, I was struck by three pieces of information related to machined Be capsules: 1) that the speed of sound (and perhaps other properties) in Be grains is markedly different in orthogonal directions, 2) that the grain size in the best refined bulk Be samples is 10 to $15 \mu \mathrm{m}$, and that these grains in the best case are randomly oriented, and 3) that we are concerned about capsule homogeneity on the level of 1 part in $10^{4}$, presumably over angular length scales corresponding to the maximum in the growth curve. It seems to me that the first two points might be inconsistent with the third, and this led me to attempt to model the effect of randomly oriented Be grains on the radially dependent properties of a capsule.

The model details and results are given below, but let me briefly summarize the spirit of the model and calculations here. I first produce a piece of Be divided into randomly oriented grains of a given average size. There is some flexibility in the breadth of the size distribution, though it seems to have little effect on the results. Each grain has an internal grain vector direction, and there is no directional correlation between neighboring grains. The value of the grain property $v$ (I was thinking about sound speed but there could be other properties) is given a value in the vector direction, $v_{\|}$, and orthogonal to this direction, $v_{\perp}$, such that a) the ratio of these values is $p$,

$$
p=\frac{v_{1}}{v_{\perp}}
$$

and b) $v_{\|}$and $v_{\perp}$ are chosen so that the grain orientation average value of $v$ is $\langle v\rangle_{g o} \equiv v_{0}$, which in the calculations I take as unity meaning that all velocities are measured in units of $v_{0}$. I then obtain an average value, $\bar{v}(\theta)$, of $v$, size-averaged over the grains a radial line traverses as one "spheremaps" around ( $\theta=0$ to $2 \pi$ ) a 2 $\mathrm{mm}$ diameter Be shell with a $150 \mu \mathrm{m}$ wall composed of this "model" material. In

This work was performed under the auspices of the U.S. Department of Energy by the University of California, Lawrence Livermore National Laboratory under Contract No. W-7405-Eng-48. 
fact the calculations are done on a planar slab (unrolled sphere?) $150 \mu \mathrm{ms}$ thick in the $z$-direction ("radial" direction) with appropriate periodic boundary conditions in the $x$ and $y$ directions. Multiple "traces" are taken on the sample material, and from these an average power spectrum of the variation in $\bar{v}$ can be determined as a function of $p$ and the grain size.

At this point $v$ is simply some orientationally dependent grain property. However, in order to convert this power spectra data to something that I can compare with NIF surface finish specifications, I compute what the inner capsule surface would look like if I were to convert the $\bar{v}$ to a length, and to do so I now associate $v$ with a shock velocity. Specifically I compute what the modulations would be in a wavefront traveling at velocity $\bar{v}(\theta)$ after traversing $150 \mu \mathrm{m}$ of material. The average time for the wavefront to travel $150 \mu \mathrm{m}$ is

$$
t_{0}=\frac{150 \times 10^{3} \mathrm{~nm}}{\langle v\rangle_{g o}},
$$

so the wavefront displacement profile at this time is given by

$$
\begin{aligned}
h(\theta) & =\left(\bar{v}(\theta)-\langle v\rangle_{g o}\right) \cdot t_{0} \\
& =\left(\bar{v}(\theta)-\langle v\rangle_{g o}\right) \cdot \frac{150 \times 10^{3} \mathrm{~nm}}{\langle v\rangle_{g o}} \\
& =\left(\frac{\bar{v}(\theta)}{\langle v\rangle_{g o}}-1\right) \cdot 150 \times 10^{3} \mathrm{~nm} \\
& =(\bar{v}(\theta)-1) \cdot 150 \times 10^{3} \mathrm{~nm}
\end{aligned}
$$

since $\bar{v}(\theta)$ is measured in units of $\langle v\rangle_{g 0}$.

Before proceeding to the detailed results it is worth asking two questions. First, what do we expect and second, how is this model relevant to implosion physics. The first question is easier. The power spectrum should be flat from low power out to modes representing the lengthscale of the grains. Since the grains have uncorrelated, random orientation, the overall "surface" rms (root of the sum of the power) should be inversely proportional to the square root of the number of random values of $v$ averaged (i.e. the number of grains along the $150 \mu \mathrm{m}$ radial line) in determining $\bar{v}$. However this number is inversely proportional to the grain size, thus we expect the surface rms to scale with the square root of the grain size. How this is manifest in the power spectrum as a function of mode number will be discussed later. The question of the relevance of these calculations to implosion physics is certainly more complicated, and will have to be answered by others. As will be shown shortly, if the full anisotropy of the sound velocity $(p \cong 1.1)$ manifests 
itself in the breakout profile then the grain size would have to be reduced to less than $0.1 \mu \mathrm{m}$ to meet specifications (assumes inner and outer surface specs are similar). This is not likely to happen. The model, of course, is a gross oversimplification in a number of ways. I can imagine that grain boundaries play a complicated role in the way shocks are transferred from grain to grain. But perhaps the most critical issue is whether for the shock amplitude and velocity in a NIF implosion, the orientationally dependent sound speed is a relevant quantity, or even if the concept of crystalline grains is relevant. Does the shock have any sense of the crystalline order or is it more like a hard wall moving through an ideal gas? Further, there appear to be questions with regard to where on the Be phase diagram we are as the first shock passes through. Because of these questions (which I'm not prepared to answer!!) I formulated the calculation in terms of the $p$ parameter, which might be thought of as parameterizing the degree of relevant anisotropy with respect to shock speed in the structure. What is clear from these model calculations is that the relevant value of $p$ had better be pretty close to unity.

\section{Model details.}

As noted above the calculations are done on a planar slab rather than a spherical shell. Given the small size of the grains and the fact that the inner and outer circumferences differ by only about $15 \%$, the flattening of the sphere (loss of curvature) should not qualitatively effect the results. Thus the system studied was a planar slab, $150 \mu \mathrm{m}$ thick $(z), 2000 \pi \mu \mathrm{m}$ long $(x)$ to represent the typical length of an equatorial AFM trace on a 2-mm shell, and $N_{t r} d_{g} \mu \mathrm{m}$ wide $(y)$, where $d_{g}$ is the average grain size and $N_{t r}$ (typically 100) is the number of "traces" taken along the $x$ direction, spaced $d_{g} \mu \mathrm{ms}$ apart. The slab is periodic in the $x$ and $y$ directions.

Grains are created in the slab as follows. For a given run the average grain size $d_{g}$ is selected. The number of grains, $N_{g}$, that will be created is determined by assuming a spherical grain volume of $(4 / 3) \pi\left(d_{g} / 2\right)^{3}$ and dividing this into the slab volume $\left(150 \times 2000 \pi \times N_{t r} d_{g}\right)$, the result being that $N_{g}=1.8 \times 10^{6} \cdot\left(N_{t r} / d_{g}{ }^{2}\right)$. In order to divide up the slab into grains, random locations are sequentially selected, and the new point is tested to make sure that it is at least some preset fraction, $f$, of $d_{g}$ away from all other points. If it is, the position of the point is stored, if not another point is randomly selected. As will be shown later, the size of the "excluded volume" parameter, $f$, has only a small effect on the both the distribution of grain sizes and their shape, larger values of $f$ lead to more symmetric grains and a narrower distribution of sizes. However these differences have essentially no effect on the computed power spectra.

Once $N_{g}$ points are placed, each grain is defined as the region of space that is closest to each point. The grain is thus the Wigner-Seitz cell defined by the perpendicular bisecting planes of the lines connecting a point with its neighbors. To 
each grain (or defining point) a random vector direction is assigned, which represents the direction of the maximum of the grain property $v$, which we denote by $v_{\|}$. The value of $v$ orthogonal to this vector is $v_{\perp}$. The assigned vector makes an angle $\theta$ with the $z$-direction. Because of my desire to characterize the anisotropy of $v$ by the ratio $p=v_{\|} / v_{\perp}$, and have the orientation averaged value $\langle v\rangle_{g o} \equiv v_{0}$, determination of the value of $v$ in the $z$ direction, $v_{z}$, for a given value of $p$ is a bit complicated, the details are given in Appendix A. The result is

$$
v_{z}=\frac{v_{\|}}{\left(p^{2} \sin ^{2} \theta+\cos ^{2} \theta\right)^{1 / 2}}
$$

where

$$
v_{\|}=v_{0} \times \frac{2\left(1-p^{2}\right)^{1 / 2}}{\ln \left[\frac{1+\left(1-p^{2}\right)^{1 / 2}}{1-\left(1-p^{2}\right)^{1 / 2}}\right]}
$$

insures that $v=\langle v\rangle_{g o}$.

In order to obtain the average value of $v$ through the slab, $\bar{v}(x, y)$, at a given $(x, y)$ position, I first identify the grain which contains the point $\left(x, y, z_{i}\right)$ by determining which selected grain point it is closest to. The average value $\bar{v}(x, y)$ is then given by

$$
\bar{v}(x, y)=\frac{1}{n_{z}} \sum_{i=1}^{n_{z}} v_{z}\left(x, y, i \times \frac{150}{n_{z}}\right)
$$

where in the calculations presented here $n_{z}=150$. Test runs with larger values of $n_{z}$ (i.e. smaller "grid" spacings for sampling) gave essentially identical results for the derived power spectra and grain size distributions as shown in Appendix B.

For a given value of $y$, the value of $\bar{v}(x, y)$ at $n_{x}=3600$ evenly spaced $x$ points $(\sim 1.75 \mu \mathrm{m}$ apart) were sampled to create a single "trace", the data being similar in format to what is collected by the Spheremapper. In Appendix B I show that taking $n_{x}=36000$ points has no effect on the result for modes of interest. As noted earlier, typically 100 evenly spaced traces (values of $y$ ), were calculated. Each was Fourier transformed, the power calculated, and the results for the collection of traces averaged to give a power spectrum in the property $v$. This is then converted to a power spectrum in the wave front displacement as described earlier (eq 3). 
In addition to the power spectra data, I also collected data about the distribution of grain sizes as I sampled along each of the $150 \mu \mathrm{m}$ long lines in the $z$-direction at each point $(x, y)$. This was done by noting the number of sequential points that were assigned to the same grain. The data presented is based upon grain identification at $1 \mu \mathrm{m}$ intervals along the $150 \mu \mathrm{m}$ thickness $\left(n_{z}=150\right)$, Appendix B shows that there are small differences in the shape of the distribution if $0.1 \mu \mathrm{m}$ intervals are used. Figure 1 shows the size distribution results for grain sizes $\left(d_{g}\right)$ of $5,10,20$, and $50 \mu \mathrm{m}$ for $f=0$ and 0.5 , as well as what one would expect to find if the grains were perfect spheres with these diameters. Note that the differences between the $f=0$ and $f=1 / 2$ grain size distributions are small. The $f=1 / 2$ condition requires the "source points" for each of the grains to be at least separated by $d_{g} / 2$, and this leads to more symmetric grains. Thus the blue curves are slightly higher at the peak, and the red curves are slightly higher at the extremes, consistent with the $f=0$ grains being a little more irregular. But clearly the differences are not great. More important is the comparison with the ideal spherical grain (green) - clearly we see that the model grains span a much broader distribution of shapes.

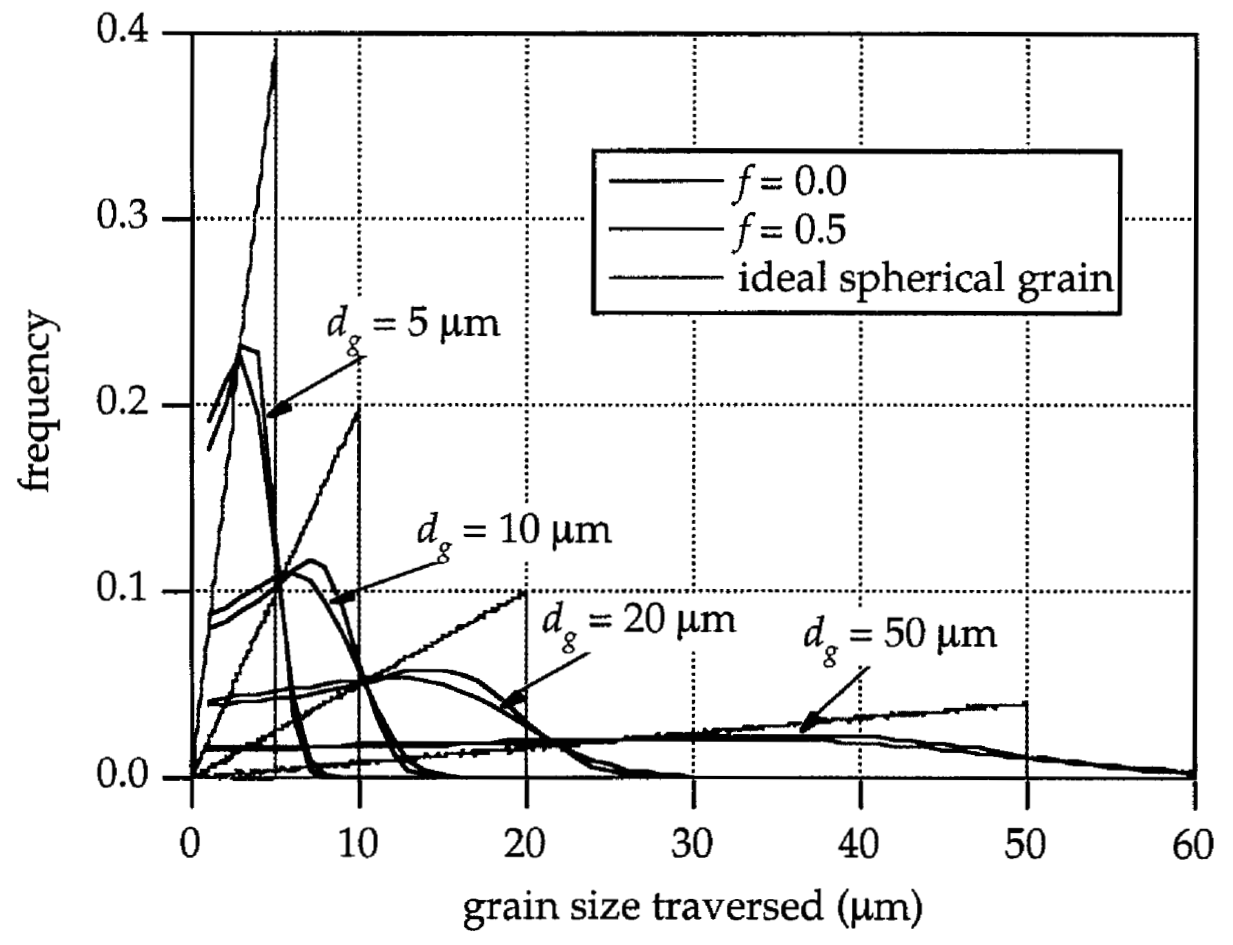

Figure 1. Shown is the distribution of grain sizes detected as one traverses the $150 \mu \mathrm{m}$ thick layer for grains of size $d_{g}$ created with $f=0$ and $1 / 2$ (red and blue). The green lines for each set are the result one would get for a spherical grain of diameter $d_{g}$.

In order to compare with a "real sample" of Be, Bob Margevicius (LANL) sent me a photomicrograph of a polished Be surface, a $\sim 300 \mu \mathrm{m}$ square patch of which is shown on the left of Figure 2. Clearly the grains are quite irregularly shaped, and 
the distribution of sizes is perhaps 5 to $30 \mu \mathrm{m}$. On the right is a similarly sized patch created from the model calculation with $d_{g}=20 \mu \mathrm{m}$. The interfaces between the grains in the model are perfectly flat, the irregular interfaces in the figure are an artifact of the graphics scheme used to create the picture. In general the model grains are a little more homogeneously sized and perhaps a little more symmetrical. But clearly aspects of the size distribution and shape seen in the micrograph are reproduced in the model. Appendix $C$ contains $100 \mu \mathrm{m}$ patches of model samples with $d_{g}=5,10,20$ and $50 \mu \mathrm{m}$.
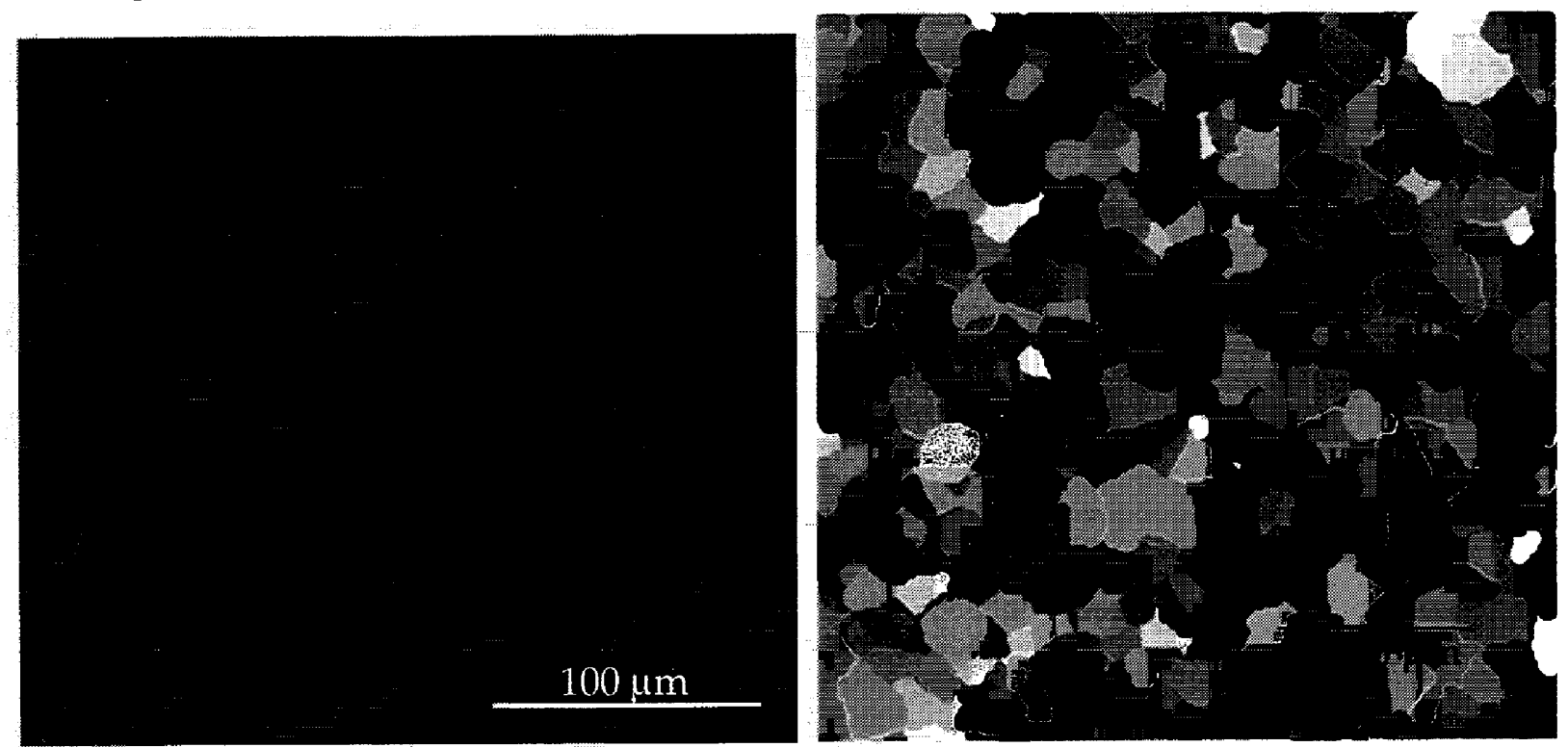

Figure 2. Left: photomicrograph of polished Be surface supplied by Bob Margevicius (LANL). Grain size is about 5 to $30 \mu \mathrm{m}$. Right: "polished" surface of a $300 \mu \mathrm{m}$ patch from the simulation, input grain size $\left(d_{g}\right)$ was $20 \mu \mathrm{m}$. The distribution of grain sizes in the two images is similar. The appearance of non-planar interfaces between model grains is an artifact of the graphics.

\section{Results.}

Power spectra information was computed for grain sizes $\left(d_{g}\right)$ of $5,10,20$, and $50 \mu \mathrm{m}$, and values of the grain anisotropy index $p$ of 1.001,1.01, and 1.1. In addition calculation were done for values of $f$ equal to $0,1 / 4$, and $1 / 2$, to see if the results were sensitive to modest differences in grain shape. The results for three sample cases shown in Figure 3 indicate they are not. A more careful examination in which the rms was calculated for each case by taking the square root of the sum of the powers shows that the $f=0$ case is between 0.2 and $2 \%$ rougher than the $f=1 / 2$ case, with no regular dependence on $d_{g}$. Because of this lack of dependence on $f$ only results for the $f=0$ case will be shown in the remainder of this section. 


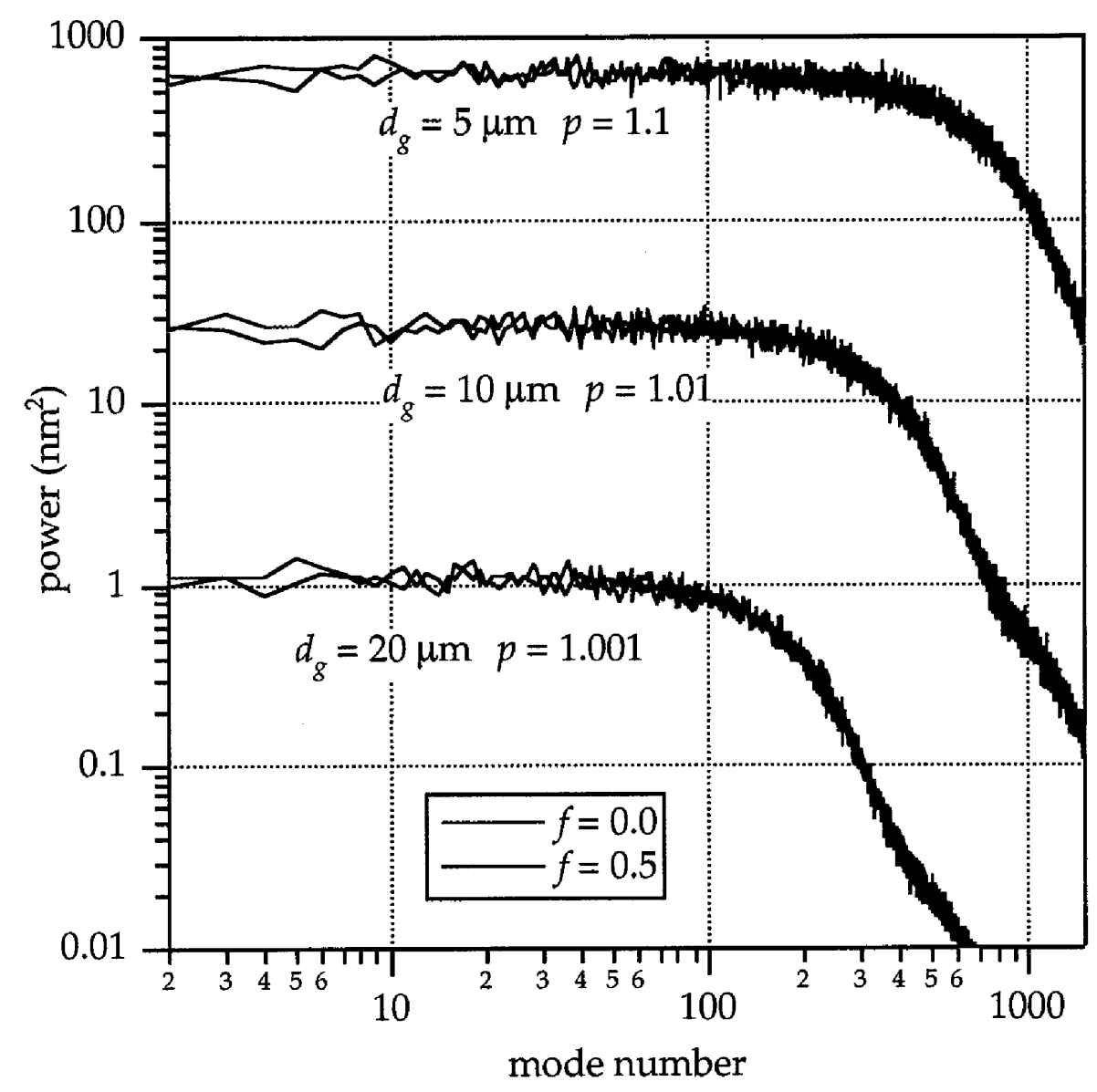

Figure 3. Shown are power spectra of three $d_{g}, p$ cases for $f=0$ and $1 / 2$. There is clearly no important $f$ dependence in the results.

The power spectra results for all the various $d_{g}, p$ parameter pairs studied are shown in Figure 4. There are several points to be made. First, the shape of the power spectra reflects the grain size. The power drop-off at large $k$ goes in each case as $k^{-3}$, and the $k$-mode at which the slope begins to go flat, $k_{t}$, roughly gives the grain size when divided into the trace length $\left(d_{g} \cong 2000 \pi / k_{t}\right)$. I haven't yet figured out the physical meaning of the $k^{-3}$ dependence, but note that it is the same as is seen in plasma polymer films. ${ }^{1}$ Second, as expected, smaller grains and smaller values of $p$ reduce the power. Third, and perhaps most important, within the confines of this simple model, grain size reduction is not going to help much in reaching the NIF design requirement (black curve) if the full asymmetry of the sound speed for $\mathrm{Be}(p \cong 1.1)$ is a relevant. Reduction in the effective anisotropy, $p$, is required. For the examples studied only $d_{g}=5 \mu \mathrm{m}$ (already smaller than current technology I think) and $p=1.001$ come close to meeting the requirement. For

${ }^{1}$ G. W. Collins, S. A. Letts, E. M. Fearon, R. L. McEachern, and T. P. Bernat, "Surface Roughness Scaling of Plasma Polymer Films," Phys. Rev. Lett. 73, 708 (1994). 


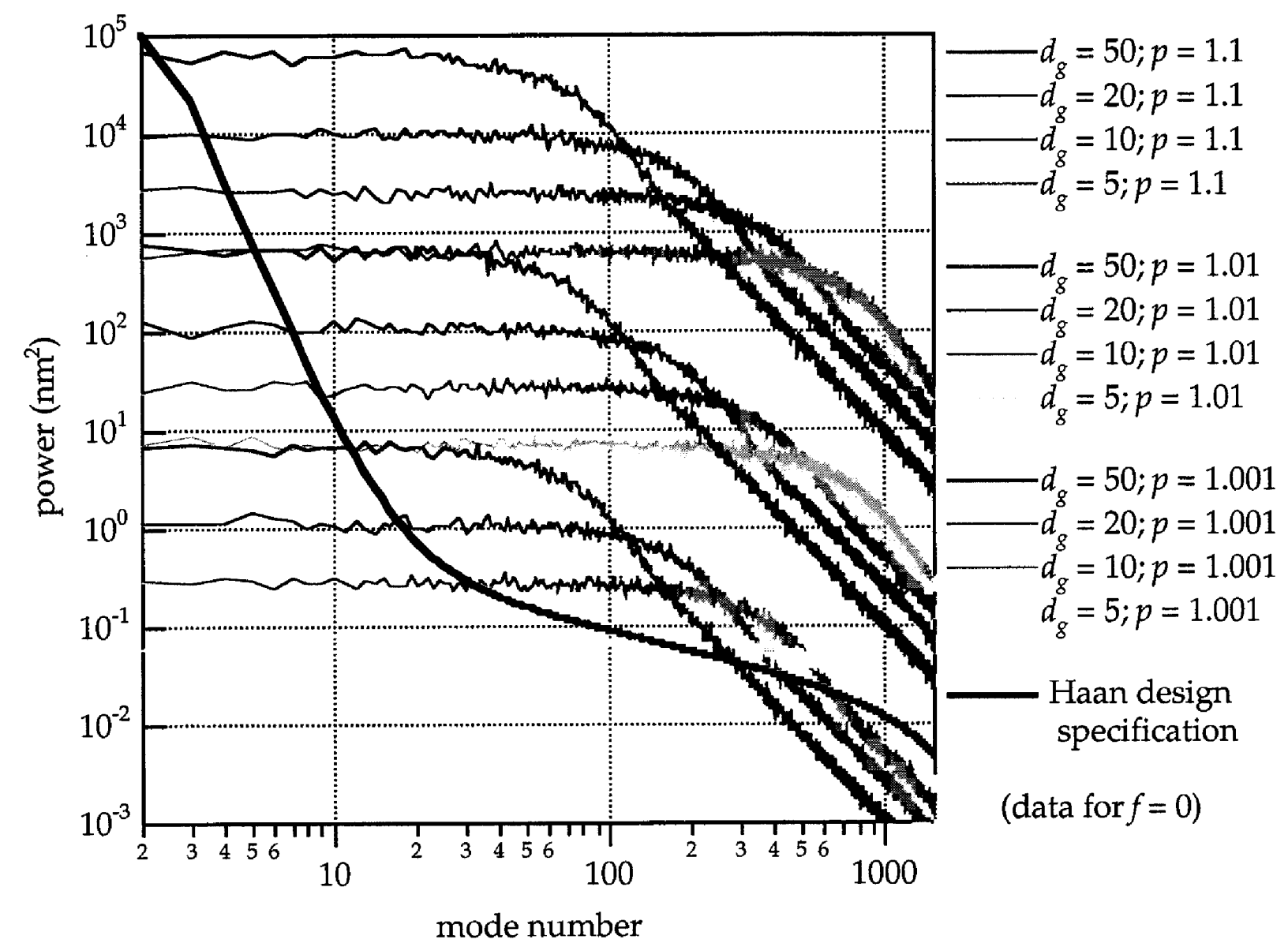

Figure 4. Shown are the power spectra for the $d_{g}, p$ parameter pairs studied, along with the NIF design specification. All results are for $f=0$.

$p=1.1$ the grain size scaling arguments presented below show that a grain size of less than $0.1 \mu \mathrm{m}$ would be necessary to meet the design specification.

As expected, the data in Figure 4 exhibit $d_{g}$ and $p$ dependent scaling. The latter is easiest to demonstrate since it simply reflects the magnitude of the effect through $(p-1)$, and not the geometry. In Figure 5 I demonstrate the scaling by plotting the power divided by $(p-1)^{2}$ as a function of $k$, the squared dependence because the power reflects the square of the amplitude. The scaling with grain size is more complicated. The actual surface rms, equal to the square root of the sum of the power over all modes, scales with the square root of $d_{g}$ as shown in Figure 6 where the rms for the $p=1.001$ case is plotted as a function of the grain size parameter, $d_{g}$. This result was predicted earlier based on simple statistical arguments. However if one examines Figure 4 in the flat sections it is clear that the power scales with $d_{g}^{2}$. This is demonstrated in the plot on the left in Figure 7, where the power divided by $d_{g}{ }^{2}$ is plotted as a function of mode number. However one can also scale the data in Figure 4 so that the sloped sections superimpose, and this is accomplished by 
multiplying the power by $d_{g}$ as is shown on the right side of Figure 7. Thus the $d_{g} 1 / 2$ scaling of the total rms represents a sort of averaging between the scaling of the flat sections (lengthscales greater than the grain size) and the $k^{-3}$ sections (lengthscales less than the grain size).

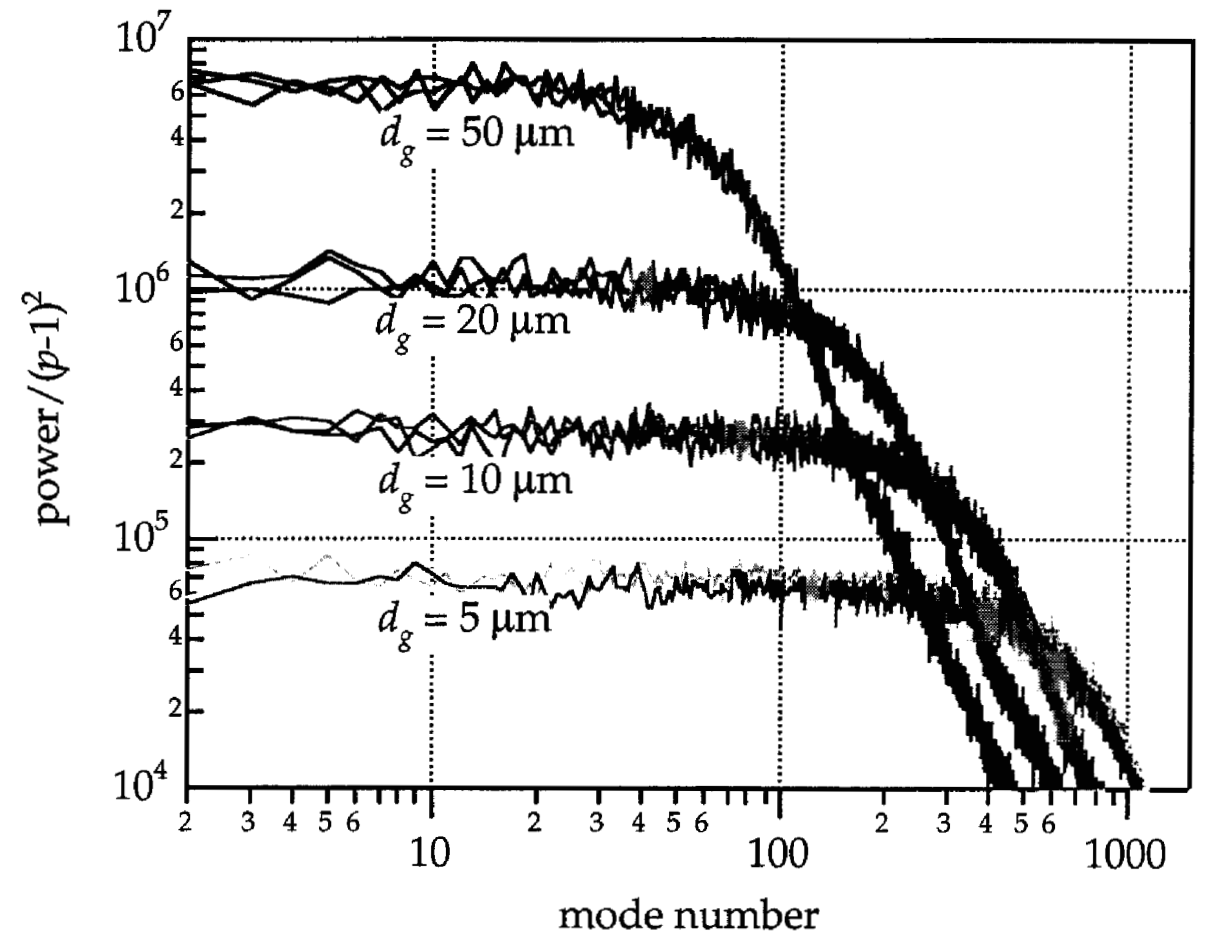

Figure 5. Demonstration of the $p$-dependent scaling.

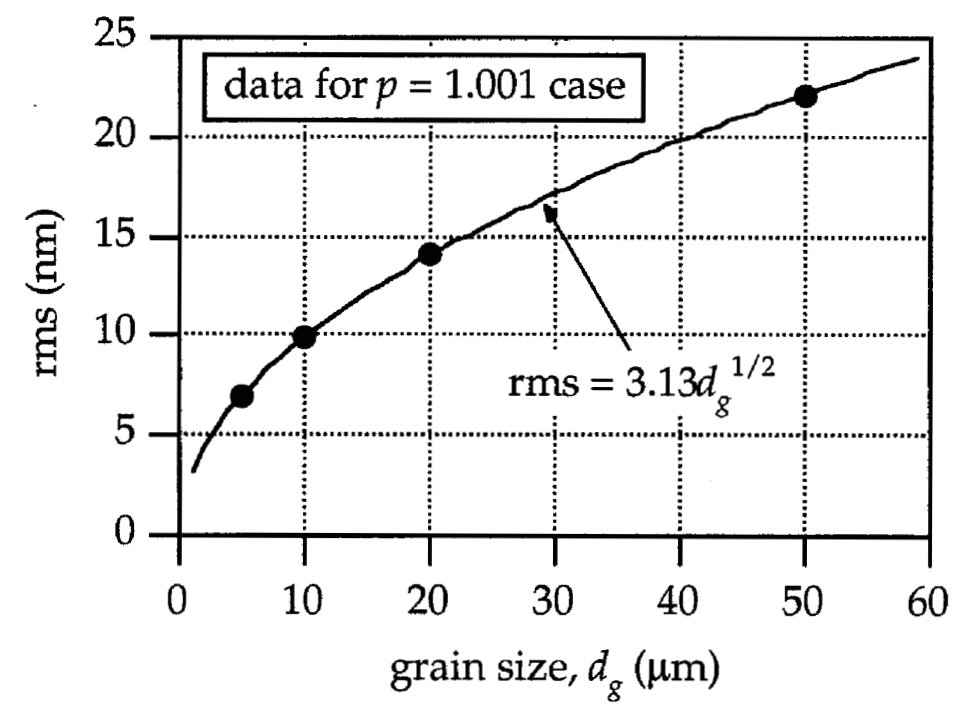

Figure 6. The computed "shock breakout" rms (square root of the sum of the power over all modes) is plotted as a function of the grain size parameter, $d_{g}$, for the $p=1.001$ case. The displayed fit shows the predicted $\sqrt{d_{g}}$ dependence. 

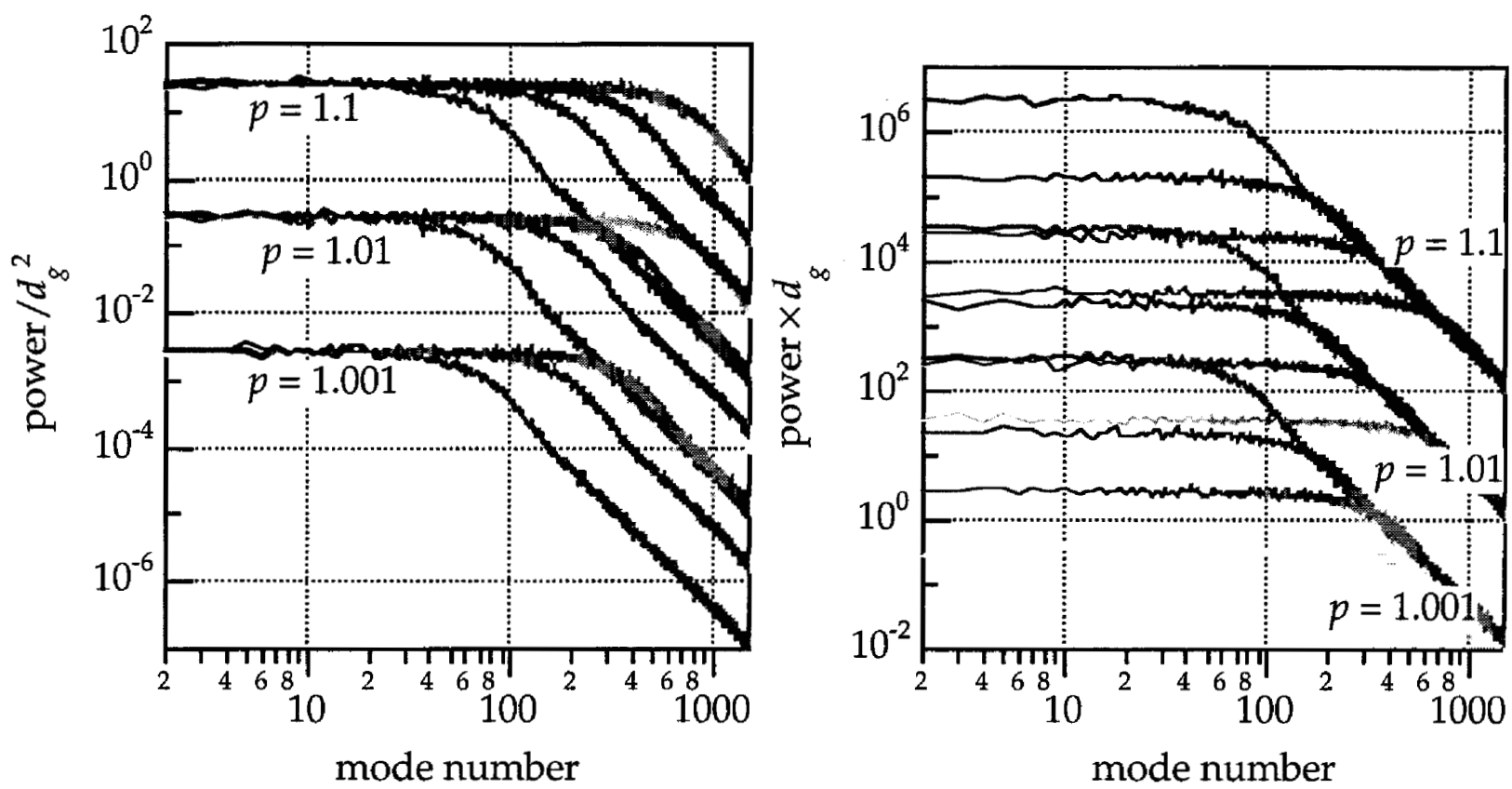

Figure 7. Two examples of the $d_{g}$ scaling of the power.

Appendix A. Determination of the value of the property $v$ for a grain tipped at an angle $\theta$ from the $z$ direction.

The value of $v$ in a direction at an angle $\theta$ to $v_{\|}$in the grain can be evaluated as follows. Imagine a grain oriented in an arbitrary reference frame so that the $v_{\|}$ direction is in the $z$ direction. The surface of the ellipsoid

$$
\left(\frac{x}{v_{\perp}}\right)^{2}+\left(\frac{y}{v_{\perp}}\right)^{2}+\left(\frac{z}{v_{\|}}\right)^{2}=1
$$

defines the value of the property $v$ as the distance from the origin to the ellipsoid surface at any spherical coordinate pair $(\phi, \theta)$, where $\theta$ is the angle from the $z$-axis (later to become the angle of grain tilt). The $x, y$, and $z$ components of $v$ are given in the normal fashion so that $x=v \sin \theta \cos \phi, y=v \sin \theta \sin \phi$, and $z=v \cos \theta$; we can rewrite the ellipsoid equations as

$$
\left(\frac{v \sin \theta}{v_{\perp}}\right)^{2}+\left(\frac{v \cos \theta}{v_{\|}}\right)^{2}=1
$$


or

$$
v=\frac{1}{\left(\left(\frac{\sin \theta}{v_{\perp}}\right)^{2}+\left(\frac{\cos \theta}{v_{\|}}\right)^{2}\right)^{1 / 2}}=\frac{v_{\|}}{\left(p^{2} \sin ^{2} \theta+\cos ^{2} \theta\right)^{1 / 2}}
$$

where we have replaced $v_{\perp}$ by $v_{\|} / p$. Note for $p=1$ that $v_{\|}=v$ as expected. The last step is to impose the condition that $\langle v\rangle_{g o} \equiv v_{0}$, this means

$$
v_{0}=\frac{\int_{0}^{\pi} v(\theta) \sin \theta d \theta}{\int_{0}^{\pi} \sin \theta d \theta}=\frac{v_{1}}{2} \int_{0}^{\pi} \frac{\sin \theta d \theta}{\left(p^{2} \sin ^{2} \theta+\cos ^{2} \theta\right)^{1 / 2}}=\frac{v_{1}}{2} \times \frac{\ln \left[\frac{1+\left(1-p^{2}\right)^{1 / 2}}{1-\left(1-p^{2}\right)^{1 / 2}}\right]}{\left(1-p^{2}\right)^{1 / 2}} .
$$

Thus for a given value of $p$ the value of $v_{\|}$is

$$
v_{\|}=v_{0} \times \frac{2\left(1-p^{2}\right)^{1 / 2}}{\ln \left[\frac{1+\left(1-p^{2}\right)^{1 / 2}}{1-\left(1-p^{2}\right)^{1 / 2}}\right]}
$$

and the value of $v_{z}$ for a grain tilted by an angle $\theta$ from the radial direction is given in analogy to eq $(\mathrm{A}-3)$ by

$$
v_{z}=\frac{v_{\|}}{\left(p^{2} \sin ^{2} \theta+\cos ^{2} \theta\right)^{1 / 2}}
$$

Appendix B. Examination of grid size effects.

The data presented in the body of the memo are based on grain identification through the slab at $1 \mu \mathrm{m}$ intervals $\left(n_{z}=150\right)$ and sampling along the length of the slab at $n_{x}=3600$ points, which represents about a $1.75 \mu \mathrm{m}$ interval. To confirm that these parameters are adequate to produce accurate data for samples of interest, a study was conducted on the $d_{g}=5 \mu \mathrm{m}$ grain size system with $f=0$, increasing $n_{z}$ to $1500\left(0.1 \mu \mathrm{m}\right.$ spacing) and increasing the sampling along the slab to $n_{x}=36000$ 
points. The $5 \mu \mathrm{m}$ grain size system with $f=0$ should be the most sensitive case of those studied since it has the smallest and most asymmetric grains.

Shown in Figure B-1 is the grain size distributions for the four cases shown in the legend. Note first that the number of sampling points, $n_{x}$, has no effect on the computed distribution since it has no effect on the grain size determination. Only the value of $n_{z}$ should matter. For $n_{z}=1500 \mathrm{I}$ have drawn a continuous curve starting at $0.1 \mu \mathrm{m}$, the smallest grain size that can be determined. The $n_{z}=150$ data appear as points at $1,2,3, \ldots \mu \mathrm{ms}$, the frequency values being scaled by a factor of 10 in order to allow comparison with the $n_{z}=1500$ data. Because the larger value of $n_{z}$ allows the detection of sub-micron grain intersections in the computation of $\bar{v}(x, y)$ that would be missed by the smaller value of $n_{z}$, the continuous curves for $n_{z}=1500$ are somewhat lower than the data for $n_{z}=150$. However the basic shape of the distribution is the same.

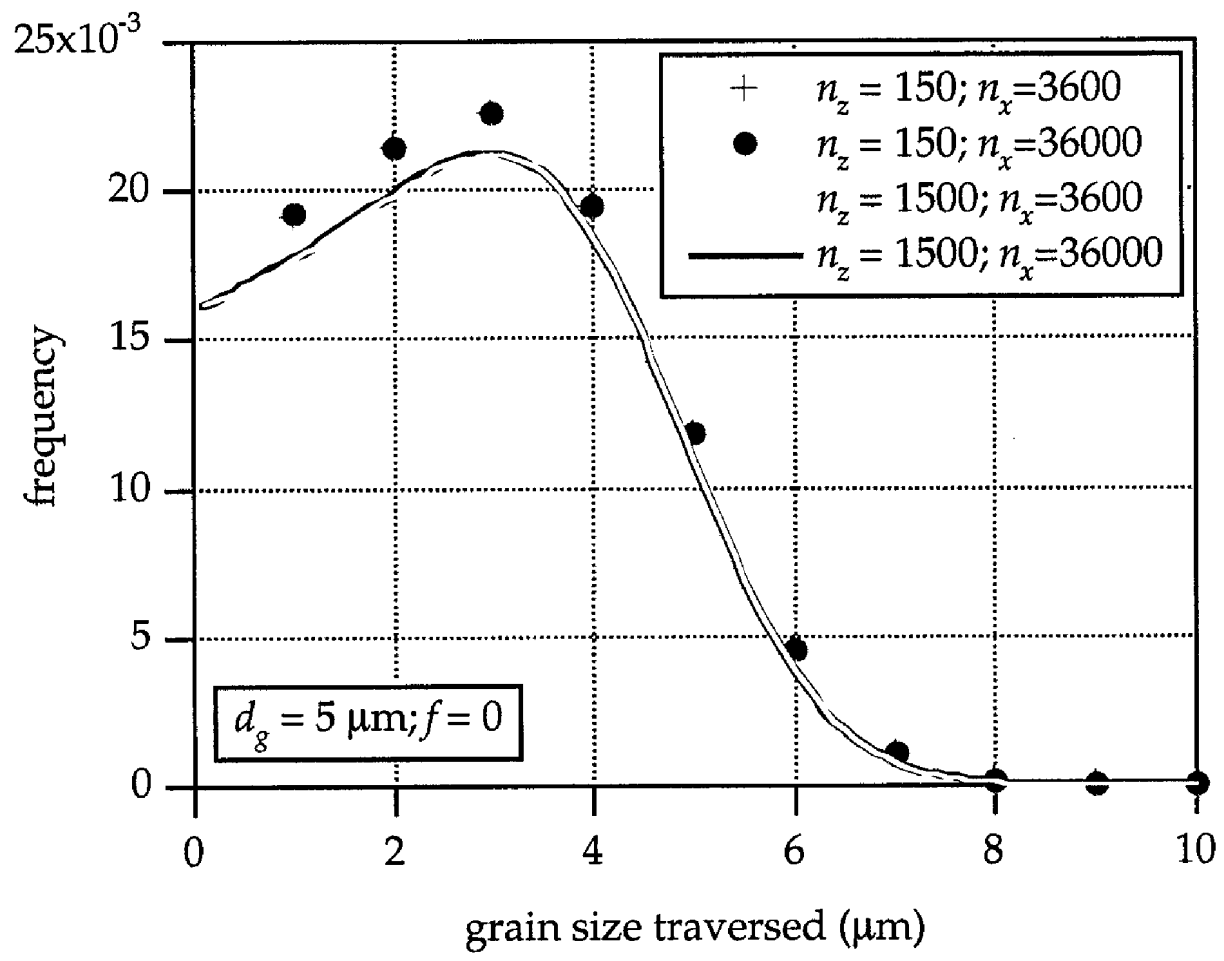

Figure B-1. Shown is the distribution of grain sizes traversed during the calculation of $\bar{v}(x, y)$ as a function of $n_{z}$ and $n_{x}$.

In Figure B-2 are shown the power spectra derived from the $\bar{v}(x, y)$ data for the four cases. For the $n_{x}=36000$ runs the computed power goes to higher mode number as expected, and in this regime (mode number $>3000$ ) there is some difference between the $n_{z}=150$ and $n_{z}=1500$ data, presumably reflecting the more accurate $\bar{v}(x, y)$ determination. But clearly for modes of interest $(<1000)$, there is no advantage to either more accurate $\bar{v}(x, y)$ determination by using a larger value of $n_{z}$ or more closely spaced $x$ sampling by using a larger value of $n_{x}$. 


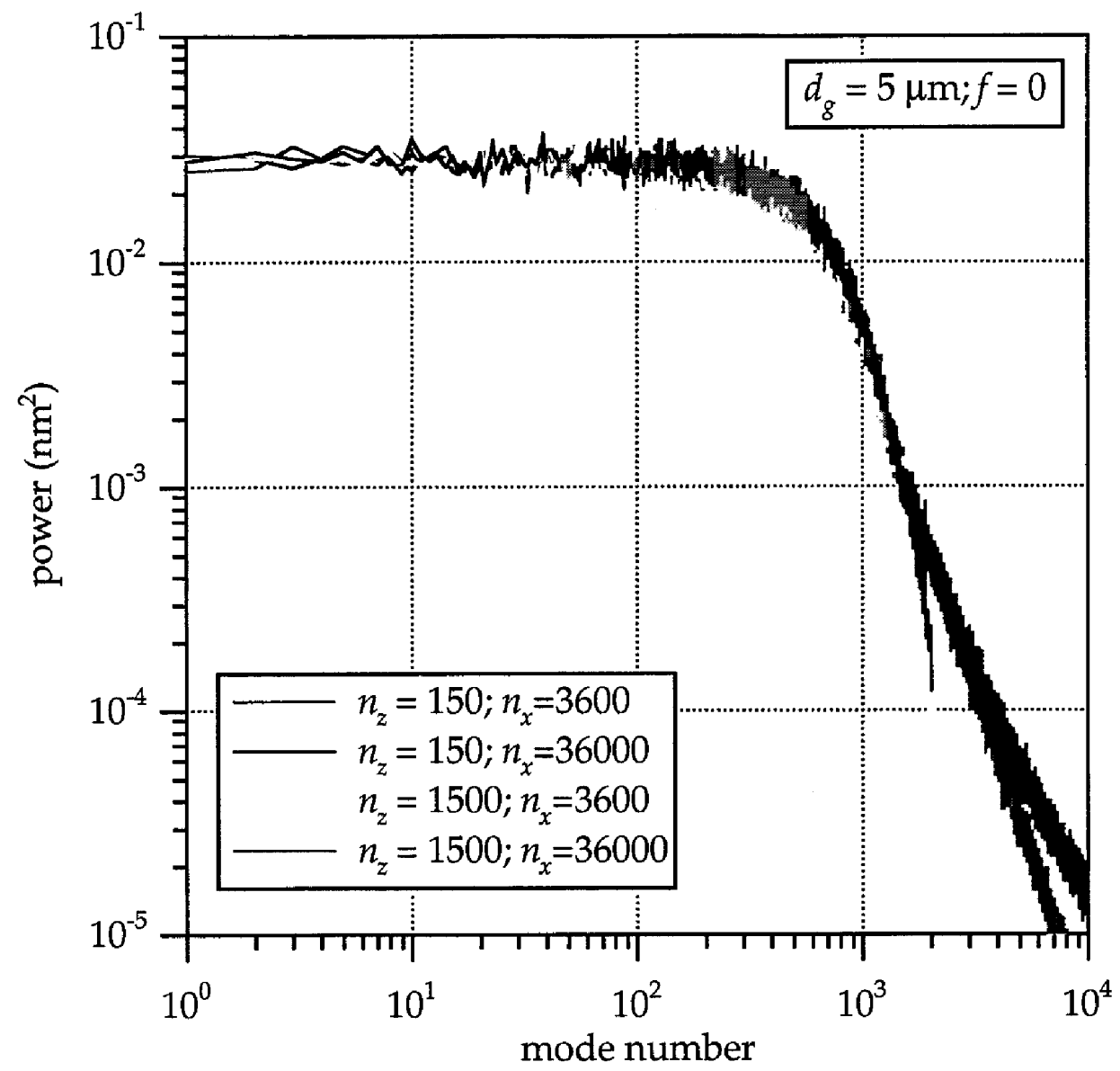

Figure B-2. Shown are the power spectra obtained as a function of the choices of $n_{z}$ and $n_{x}$. 
Appendix C. Examples of model $100 \mu \mathrm{m}$ patches.
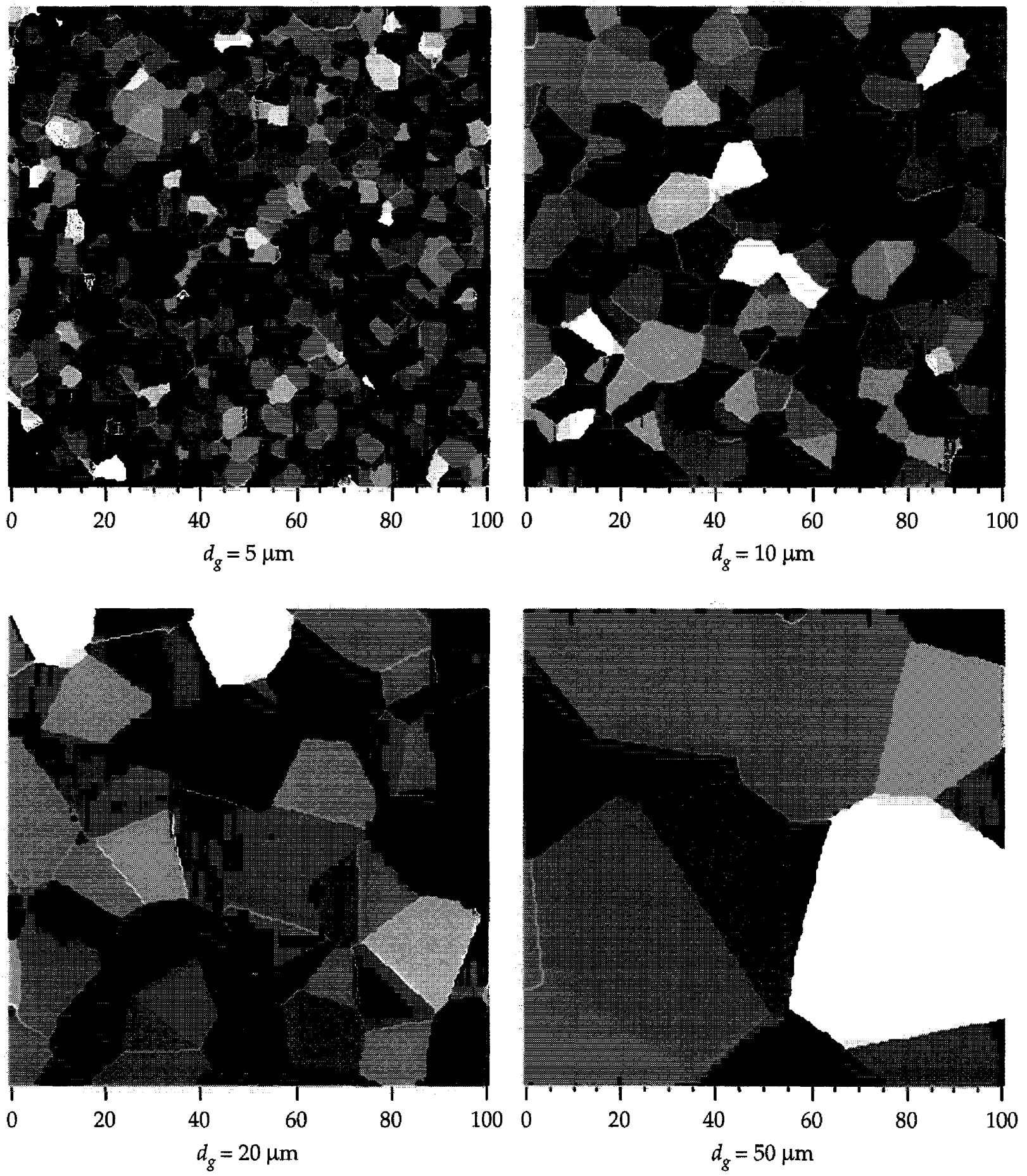

Figure C-1. Shown are $100 \mu \mathrm{m}$ square patches of "polished" model Be grain structures with $d_{g}=5,10,20$, and $50 \mu \mathrm{m}$. The interfaces between grains are in fact perfectly flat, the "roughness" seen in the figures is a graphics artifact. 
University of California

Lawrence Livermore National Laboratory

Technical Information Department

Livermore, CA 94551

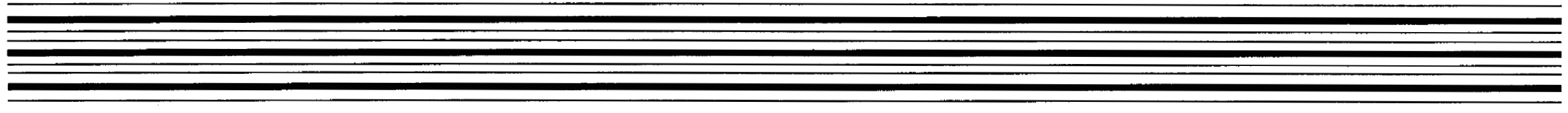

